\begin{tabular}{|c|c|c|}
\hline Beitr. Ent. & Keltern & ISSN 0005-805X \\
\hline $54(2004) 1$ & S. $75-88$ & 28.05 .2004 \\
\hline
\end{tabular}

\title{
Taxonomische Studien zur Hydrophiliden-Gattung Enochrus Thomson, 1859. - 6. Teil: Die afrikanischen Arten der Untergattungen Lumetus ZAITZEV, 1908 und Enochrus s. str.
}

\section{(Coleoptera, Hydrophilidae)}

Mit 11 Figuren

FRANZ HEBAUER

Summary

The subgenera Lumetus $Z_{A} I T Z E V, 1908$ and Enocbrus s. str. are discussed. A key to all known species of these groups is given and the male genitalia are illustrated.

Key words

Insecta, Coleoptera, Hydrophilidae, Enocbrus, systematics, taxonomy, Africa, key, maps.

\section{Zusammenfassung}

In Weiterführung der taxonomischen Studien zur Hydrophiliengattung Enocbrus THOMSON werden im vorliegenden 6 . Teil die Untergattungen Lmmetus ZAITZEV und Enocbrus s. str. vorgestellt und durch weitere Nachweise sowie 2 Bestimmungstabellen ergänzt.

\section{Einleitung}

Die in der Paläarktis durch zahlreiche Arten vertretene Untergattung Lumetus ZAITZEV ist in Afrika nur spärlich vertreten und besiedelt dort fast ausschließlich den paläarktischen Norden des Kontinents. Von E. segmentinotatus KUWERT und E. ater KUWERT liegen auch Funde aus Westafrika (Gambia, Kenia, Senegal) vor,

Ähnlich sind aus der Untergattung Enocbrus bisher nur zwei Arten (mediastinus, pellax) aus dem tropischen Afrika bekannt, eine weitere Art (melanocepbalus) zählt zur paläarktischen Fauna und ist bisher lediglich im nordöstlichen Teil des Kontinents nachgewiesen. 


\section{Danksagung und Abkürzungen}

Allen Institutionen und Kollegen, die bereit waren Material für die vorliegende Arbeit zur Verfügung zu stellen sei an dieser Stelle herzlich gedankt.

$\begin{array}{ll}\text { CBB } & \text { Coll. M. Balke, Berlin, } \\ \text { CBO } & \text { Coll. K. G. Bernhardt, Osnabrück, } \\ \text { CHG } & \text { Coll. F. Hebauer, Grafling, } \\ \text { NHM } & \text { Natural History Museum (British Museum) London (S. Hine), } \\ \text { MHNG } & \text { Muséum national d'Histoire naturelle, Geneve (I. Loebl), } \\ \text { MNHP } & \text { Muséum national d'Histoire naturelle, Paris (Y. Cambefort), } \\ \text { NMB } & \text { Naturhistorisches Museum Basel (M. Brancucci), } \\ \text { NME } & \text { Naturtkundemuseum Erfurt (M. Hartmann), } \\ \text { NMW } & \text { Naturhistorisches Museum Wien (M. A. Jäch, S. Schödl), } \\ \text { ZML } & \text { Zoological Museum Lund, Sweden (R. Danielsson). }\end{array}$

\section{Systematik}

Die Gattung Lumetus ZAITZEV, 1908 ist nach dem heutigen Genus-Typ Enochrus fuscipennis THOMSON, 1859 („Hydrophilus melanocephalus“ sensu FARBRICIUS, 1801, nec OLIVIER, 1792) definiert als eine Gruppe von Enochrus-Arten, deren letztes Glied der Maxillarpalpen kürzer ist als das vorletzte Glied, mit deutlicher lateraler Punktellipse auf dem Pronotum versehen und mit apikal nicht ausgerandetem Analsternit.

Jedes dieser Merkmale tritt nach heutiger Erfahrung einzeln auch in anderen Untergattungen von Enochrus auf: Ein verkürztes Palpen-Endglied besitzen nahezu alle Arten der Untergattung Metbydrus; eine laterale Punktellipse ist besonders deutlich bei E. (Methydrus) besperidum SHARP zu beobachten; die fehlende apikale Ausrandung des Analsternits ist sowohl bei Enoclorus s. str. einschließlich des E. pellax BALFOUR-BROWNE zu finden.

Untergattungen sind immer als Idealkategorien und als Hilfskonstruktionen zu betrachten; sie zeigen in der Regel Überschneidungen in einzelnen Merkmalen.

Die Untergattung Enochrns s. str. wurde von THOMSON (1859) auf dem heutigen GenusTyp E. melanocephalus OLIVIER, 1792 („Hydrophilus bicolor" sensu PAYKULL, 1798, nec FABRICIUS, 1792) monotypisch errichtet und durch die Maxillarpalpen mit gleichlangem 3. und 4. Glied definiert. Das Analsternit ist apikal nicht ausgerandet, das 2. Glied der Maxillarpalpen ist bei E. melanocephalus OL. fast gerade, ähnlich wie bei Cymbiodyta sowie den groBen Formen der Untergattung Metbydrus. Insgesamt sind die Maxillarpalpen dieser Untergattung in der Regel relativ kurz und gedrungen.

Die Arten dieser Untergattung zeigen eine größere Variationsbreite der einzelnen Merkmale und Überschneidungen. So hat E. pellax eine niedrige, bogenförmige Mesosternallamelle ohne Zähnchen. Bei einigen Methydms-Arten der meracus-Gruppe ist das Endglied der Maxillarpalpen kaum kürzer als das vorletzte Glied. Es sind besonders bei letzterer Gruppe alle Übergänge in Bezug auf dieses Merkmal zu beobachten. 


\section{A. Untergattung Lumetus ZAITZEV, 1908}

\section{Bestimmungstabelle}

1 Klauen auffallend lang, sensenförmig; 5,3-7,2 mm...... E. falcarius HEBAUER, 1991 Klauen von normaler Größe ....................................................................... 2

2 Oberseite blaßgelb, Maxillarpalpen und Tibien einfarbig gelb. ................................ 3

- $\quad$ Oberseite dunkel, 2. Glied der Maxillarpalpen und Tibien meist angedunkelt. ........ 4

3 Breiter oval; Pubeszenz mittlere und hintere Femora bei den Männchen 4/5, bei den Weibchen 5/6 der Schenkellänge bedeckend; Innenrand der Parameren bei ventraler Ansicht nicht winklig vorspringend; durchschnittlich größer, 5,0-7,7 mm. ........

E. bicolor (FABRICIUS, 1792)

- Schlanker oval; Pubeszenz mittlere und hintere Femora bei den Männchen 3/4, bei den Weibchen 4/5 der Schenkellänge bedeckend; Innenrand der Parameren bei ventraler Ansicht deutlich winklig vorspringend; durchschnittlich kleiner, 5,5-6,5 $\mathrm{mm}$

E. segmentinotatus (KUWEERT, 1888)

4 Elytren fein, sehr dicht punktiert, mehr seidenglänzend, pechbraun; laterale Punktellipse des Pronotum undeutlich grob und unregelmäßig; 5,5-6,0 mm

E. ater (KUWERT, 1888)

Elytren sehr fein bis obsolet, lockerer punktiert, lackglänzend, pechschwarz; laterale Punktellipse des Pronotum sehr deutlich und regelmäßig; 5,5-6,5 mm.

E. politus (KÜSTER, 1849)

\section{Die Arten}

\section{Enochrus (Lumetus) ater (KUWERT, 1888)}

1888 Phitydrus (i.sp.) ater KUNERT, p. 289 (279) (valid sp.: BAIfOUR-Browne, 1951:215) (Ägypten).

\section{Locus typicus: „Ägypten“.}

Nachweise: Afrika: Gambia: Bathurst, Jan.1968, leg. Palm (ZML, CHG); Senegal: Nat. Park Djoudj, II.1988, leg. H. Terlutter (CHG); Kenia: Tsavo Nat.R., Mtito, Andej, 900 m, I.1990, Werner leg. (CHG).- Sinai: Ayun Musa, 1.4.1989, leg. M. Balke (CBB); Oman: N Sama il 400 m, Quaylah, 21.4.1985, 23/21N-58/03E, leg. C. Holzschuh (CHG); Khor Salalah, $16^{\circ} 50,8^{\prime} \mathrm{N}-54^{\circ} 04,2^{\prime} \mathrm{E}$, light, 3.12.1997, leg. Gallagher \& Harrison (CHG, NMB).- Saudi Arabien: Dammam, 18.5.1976, leg. Büttiker (NMB, CHG).

Diagnose: Dunkler und dichter punktiert als E. bicolor, heller und kräftiger punktiert als E. politus, doch von ähnlichem Habitus und auch weitgehend sympatrisch, wurde diese Art lange Zeit verkannt, obwohl in Südosteuropa weit verbreitet und sehr häufig anzutreffen; nach Westen bis Österreich vorhanden. 
Kurzbeschreibung: 5,5-6,0 mm.- Gleichmäßig oval, stark gewölbt, pechbraun mit breit, diffus rötlich aufgehellten Seiten von Pronotum und Elytren. Kopf mit breiten Präokularmakeln. Oberseite mäßig fein, doch sehr dicht punktiert, seidenglänzend; die 3 unregelmäßigen Reihen gröberer Punkte der Elytren deutlich; laterale Punktellipse des Pronotum sehr undeutlich und unregelmäßig. Maxillarpalpen mit meist angedunkeltem 2. Glied und apikal geschwärztem Endglied (Fig. 8e). Tibien meist angedunkelt, Tarsen immer hell. Mesosternallamelle hoch, vorne senkrecht abfallend, dahinter geradlinig verlaufend (Fig. 9d). Aedoeagus ähnlich dem von E. quadripunctatus, wobei der Medianlobus die Ventralplatte nur wenig überragt (Fig. 5).

Verbreitung: Paläarktis; Afrika (Algetien, Ägypten, Sinai, Kenia, Gambia, Senegal).Arab. Halbinsel: Saudi Arabien (Nord); Oman.

\section{Enochrus (Lumetus) bicolor (FABRICIUS, 1792)}

1787 Dytiscus chrysomelinus FABRICIUS, p.192 (Deutschland).

1792 Hydropbilus bicolor FABRicius, p.184 (Dänemark).

1802 Hydrophilus fuluus MARSHAM, p. 408 («Britannia»).

1827 Hydrobius grisescens GYLLENHAL, p. 276 (Schweden).

1849 Hydrobius ferrugineus KÜSTER (Frankreich).

1853 Pbillydrus maritimnts THOMSON, p. 51 (Schweden).

1888 Philydrus Sablbergi Kuwert, p. 286 (Russland).

1888 Pbibutrus atricomis KUwERT, p. 286 (Spanien).

1888 Philydrus mnguidebilis KLwERT, p. 287 (276) (Russland, „Sarepta“).

1888 Pbitydrus sternospina KUwERT, p. 288 (277) („Bavaria Austria“).

1890 Philydrus flauts KLWERT, p. 43 (Portugal).

1892 Pbillyydrus levanderi SAHLBERG, p. 225.

1900 Philbydrus mediterraneus SAHLBERG, p. 185 (Italien).

1994 Enocbrus jukinoae MATSUI, p. 218 (Japan).

Locus typicus: Dänemark („Dania“).

Nachweise: Paläarktis. Afrika: Afr. bor. Behiti Benariye Oasis, $383 \mathrm{~km}$ SWW Cairo, 4.4.1989, leg. M. Balke (CBB, CHG).- Ägypten: Sinai, Ayun Musa, 1.4.1989, leg. M. Balke (CBB); El Fayoum, Lake Qarun, 1.12.1993, leg. Bernhardt(CBO); Marokko: 27.8./ 25.3.1983, leg. El Alaloui (CHG).- Weitere Nachweise s. SCHÖDL (1998).

Diagnose: Siehe Hebauter (1989); HeBAUER \& KLAUSNitzer (1998); LOHSE (1971), SCHÖDL (1998) Aedoeagus (Fig. 1). Maxillarpalpen (Fig. 8a). Mesosternallamelle (Fig. 9a). Die Trennung des Artenpaares bicolor- segmentinotatus ist bei SCHÖDL (1998) ausführlich dargestellt.

Verbreitung: Paläarktis; Afrika (Algerien, Ägypten, Äthiopien, Kanar. Ins., Marokko, Tunesien). 


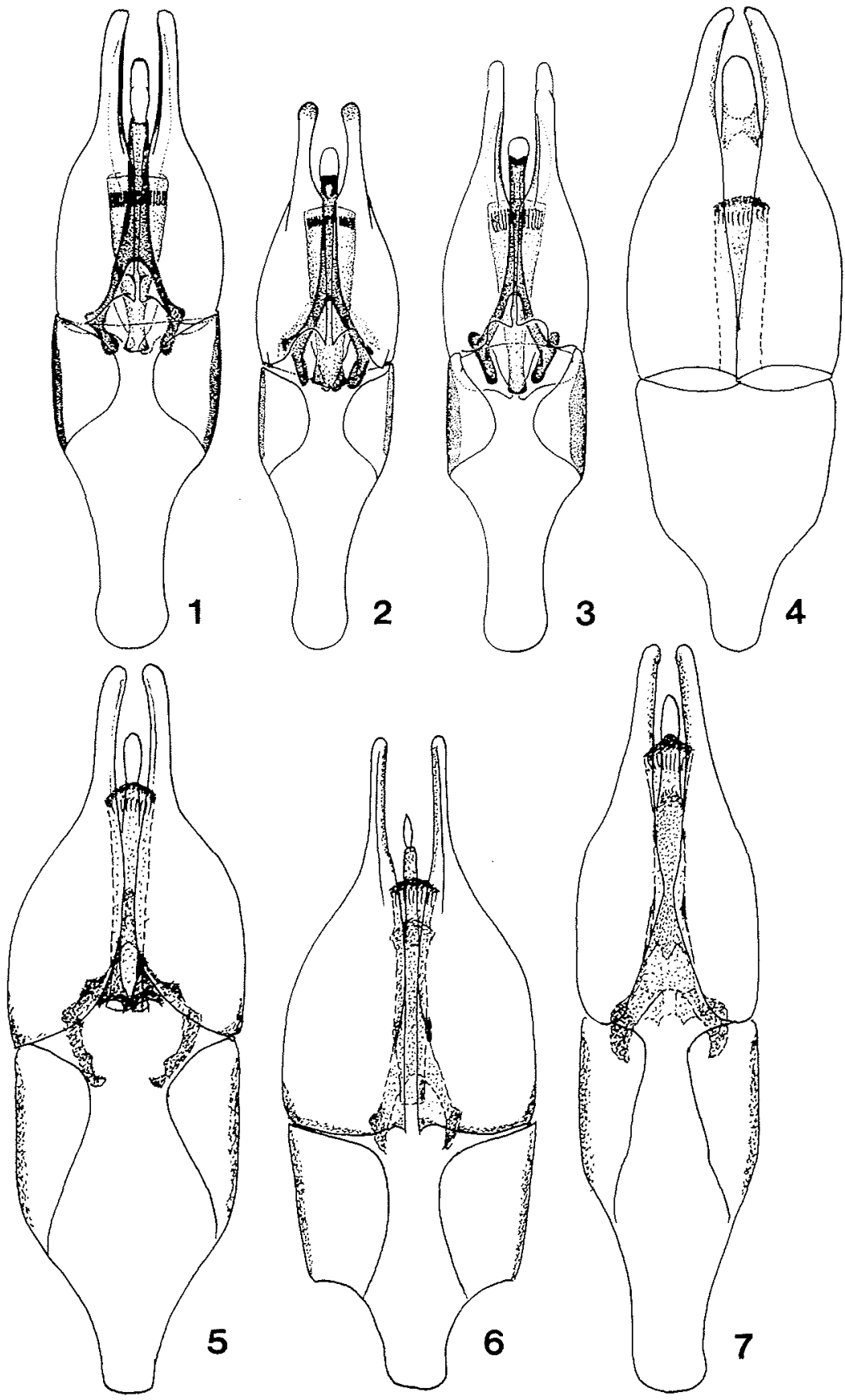

Fig. 1-7: Enochrus spp. - Adoeagus. 1. E. bicolor (FABRICIUS), 1,86 mm; n. SCHÖDL, 1998); 2. E. segmentinotatus (KUWERT), 1,57 mm; n. SCHÖDL, 1998; 3. E. falcarius HEBAUER, 1,71 mm; 4. E. mediastinus (n. BALFOUR-BROWNE); 5. E. ater (KUWERT), 1,40 mm; 6. E. melanocephalus (OlIVIER); 7. E. politus (KÜSTER); $1,40 \mathrm{~mm}$. 

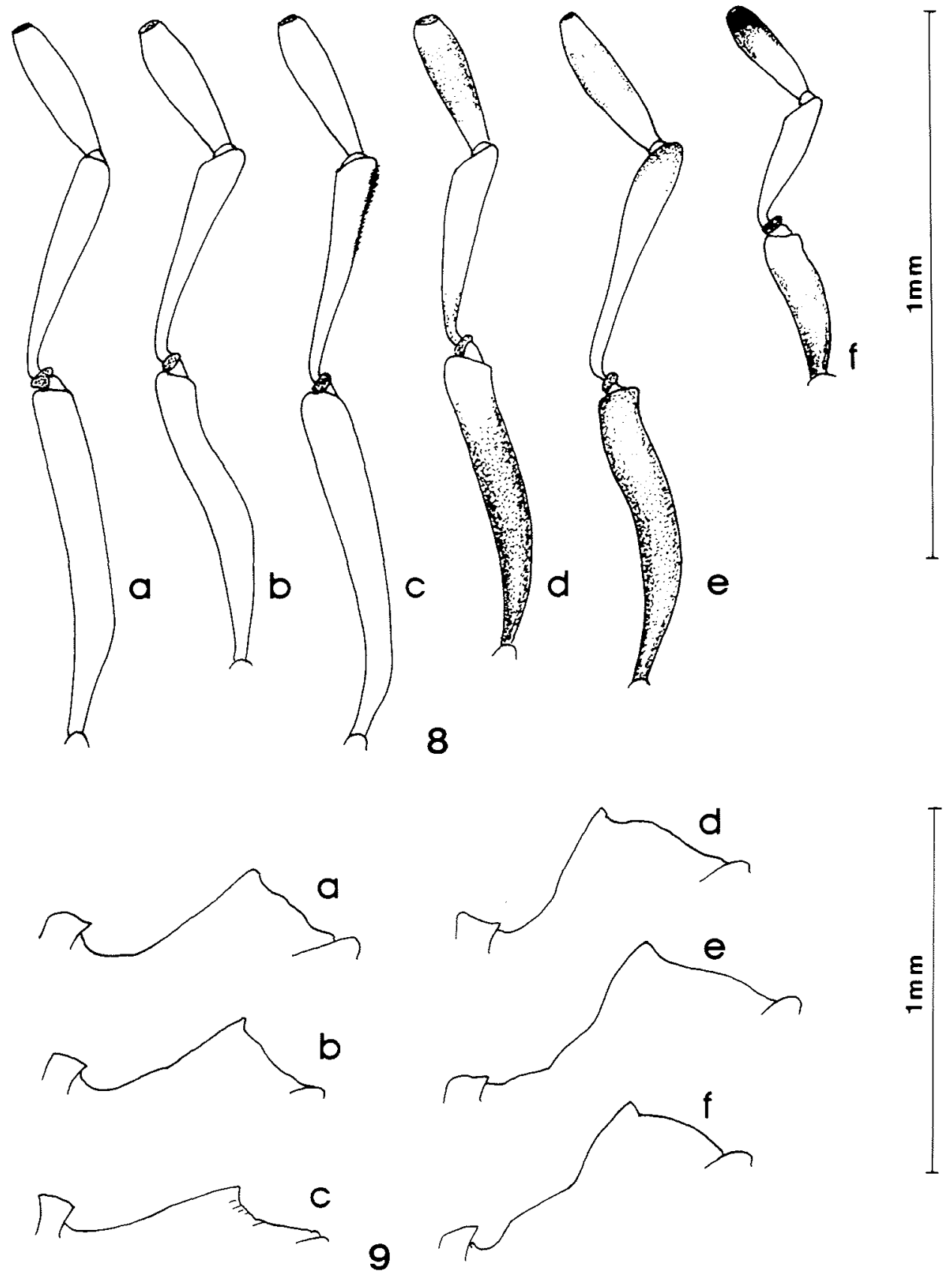

9

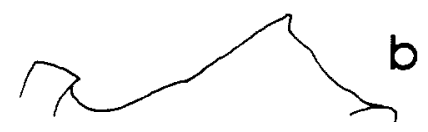

Fig. 8: Enochrus spp. - Maxillarpalpus, links: a) E. bicolor; b) E. segmentinotatus; c) E. falcarins; d) E. politus; e) E. ater; f) E. melanocephalus. - Fig. 9: Enochrus spp. - Mesosternallamelle: a) E. bicolor (n. SCHÖDL, 1998), b) E. segmetinotatus (n. SCHÖDL, 1998); c) E. falcarizs (n. SCHÖDL, 1998); d) E. ater; e) E. politus; f) E. melanocephalus. 


\section{Enochrus (Lumetus) falcarius HEBAUER, 1991}

1991 Enoclums (Lumetus) falcarius HEBALER, p. 83 (Italien).

Locus typicus: Villarmosa, Quelle in C. Castello (Sizilien).

Typenmaterial: (CHG): Holotypus (männlich): Sizilien, Villarmosa; Paratypen: Sizilien: Villadoro, Villarmosa (s. HEBAUER, 1991, SCHÖDL, 1998).

Nachweise: Tunesien: $1 \mathrm{~km}$ S Fernana, leg. Schödl (9), 3.8.1991 (CHG, NMW).

Diagnose: Siehe HEBAUER (1991); HERNANDO, et al. (1997); SCHÖDL (1998).

Tunesische Funde weichen von den italienischen und spanischen Tieren in Größe und Skulptur deutlich ab: 5,3-7,2 mm; Oberseite glänzend, gelblich (bis schwarz), dicht punktiert; Stirn und Mitte des Clypeus schwarz. Maxillarpalpen lang und schlank (Fig. 8c). Mesosternallamelle ziemlich flach mit großem Zähnchen hinter der Mitte (Fig. 9c). Aedoeagus ähnlich dem von E. bicolor; wobei der Medianlobus die Ventralplatte weit überragt (Fig. 3).

Verbreitung: Westmediterran (Italien, Spanien, Tunesien).

\section{Enochrus (Lumetus) politus (KüsTER, 1849)}

1849 Hydrabius politus KÜSTER.

1853 Philydmis atlanticus BLANCHARD, p. 51 (Teneriffa).

1870 Pbilhydus Agrigentims ROTTENBERG, p. 22 (Italien).

1879 Philbydrus sabariensis FAIRMAIRE, p. 160 (Algerien).

1885 Philydrus Cossyrensis REY, p. 260 (Sizilien).

Locus typicus: Spanien.

Nachweise: Afrika: Äthiopien: El Fayoum, Nazia, 1.12.1989, leg. Bernhardt (CBO); (weitere Belege im ZML, vidi); Kanar. Inseln: La Gomera, Bco. de Arure, 16.5.1992, leg. H. Bußler (CHG); Canary Isl. Tenerife Adeje, 26.12.1981, leg. L. Huggert (CHG); (Belege aus Teneriffa im MHNG, vidi); Tunesien: Tamerza, Neguet-Berge, 11.3.1997, leg. J. Schmidl (CHG); Qued El Magroun, 25.5.1981, leg. H. Malicky (CHG).- Marokko: 21 km NE Casablanca, 2.6.1974, leg. Borys Malkin (CHG); Marokko, VII. 1983/27.8.1983/4.5.1983, leg. El Alaloui (CHG); M.A. Ouzaid, 30.12.1992, leg. Atndt \& Gröger (NME).

Diagnose: Nachbarart von E. ater und mit diesem in der Vergangenheit oft verwechselt, da auch gebietsweise sympatrisch verbreitet; doch durch die meist viel feinere Punktierung der Oberseite, den stärkeren Glanz und die tiefschwarze Färbung der Oberseite ist die Art unterscheidbar. Die Schwärzung der Tibien und des 2. Gliedes der Maxillarpalpen ist bei E. politus konstant und deutlich vorhanden, bei $E$, ater nicht immer ausgeprägt. 
Kurzbeschreibung: 5,5-6,5.- Oval, mäßig gewölbt, lackglänzend, tiefschwarz mit breiten hellen Seiten von Pronotum und Elytren. Oberseite sehr fein bis obsolet punktiert; die 3 unregelmäßigen Reihen gröberer Punkte der Elytren deutlich. Die laterale Punktellipse des Pronotum sehr deutlich und regelmäßig. Unterseite schwarz mit hellen Knien. Mesosternallamelle hoch, vorne senkrecht abfallend, dahinter leicht konvex verlaufend (Fig. 9e). Tibien und das 2. Glied der Maxillarpalpen meist stark geschwärzt (Fig. 8d). Aedoeagus vom E. bicolor -Typ, bei welchem der Medianlobus die Ventralplatte weit überragt (Fig. 7 ).

Verbreitung: Paläarktis. Afrika: Algerien, Kanarische Inseln, Ägypten, Madeira, Marokko, Tunesien. Arabien: Oman.

\section{Enochrus (Lumetus) segmentinotatus (KUWERT, 1888)}

1888 Philydus segmentinatatus KUWERT, p. 284 (276).

1888 Pbilydrus wultur KUVERT, p. 283 (276) (Russland).

1888 Philydrus maculiapex KuwerT, p. 284 (276) (Ägypten).

1888 Pbibdrus apicinotus KuWERT, p. 285 (276) (Bagdad).

1900 Philbydrus mediterraneus var. transcaspica SAHLBERG, p. 185 (Turkmenistan).

Locus typicus: Istanbul ("Konstantinopel”) oder Griechenland ("Balcan").

Typenmaterial: Lectotypus, männlich, "Balcan"; Paralectotypen: 3 Ex. "Balcan", 1 Ex.: "Graecia Möschler"; 1 Ex.: "Thessalia Volo Stussiner" (alle MNHP). Siehe auch: SCHÖDL (1998).

Nachweise: Paläarktis. Afrika: Gambia: Bakau, 6.-26.11.1984, leg. T. Palm (ZML, CHG); S Kalili, 24.5.1981, leg. H. Malicky (CHG).- Marokko: 23.5.1983, leg. El Alaloui (CHG).- Tunesien: Djerba, 20.-26.6.1983, leg. E. Heiss (CHG); S Kabili, 24.5.1961, leg. H. Malicky (CHG); Qued El Magroun, 25.5.1961, leg. H. Malicky (CHG).- Weitere Nachweise: s. SCHÖDL (1998).

Diagnose: Siche Hebauer (1989); Hebauer \& KLAUSNitzer (1998); LohSE (1971), SCHÖDL (1998).

Diese für lange Zeit verkannte und für E. bicolor gehaltene Art wurde von SCHÖDL (1998) als speciespropria erkannt und einer gründlichen Differentialdiagnose unterzogen. Die Hauptunterschiede zu E. bicolor liegen im schlankeren Habitus, in der Bildung der männlichen Vorderklauen, in der Pubeszenz der Metafemora und in der Form der Parameren.

Aedoeagus (Fig. 2). Maxillarpalpen (Fig. 8b). Mesosternallamelle (Fig. 9b).

Verbreitung: Paläarktis. Afrika (Algerien, Ägypten, Libyen, Marokko, Tunesien, Gambia); Saudi Arabien. 


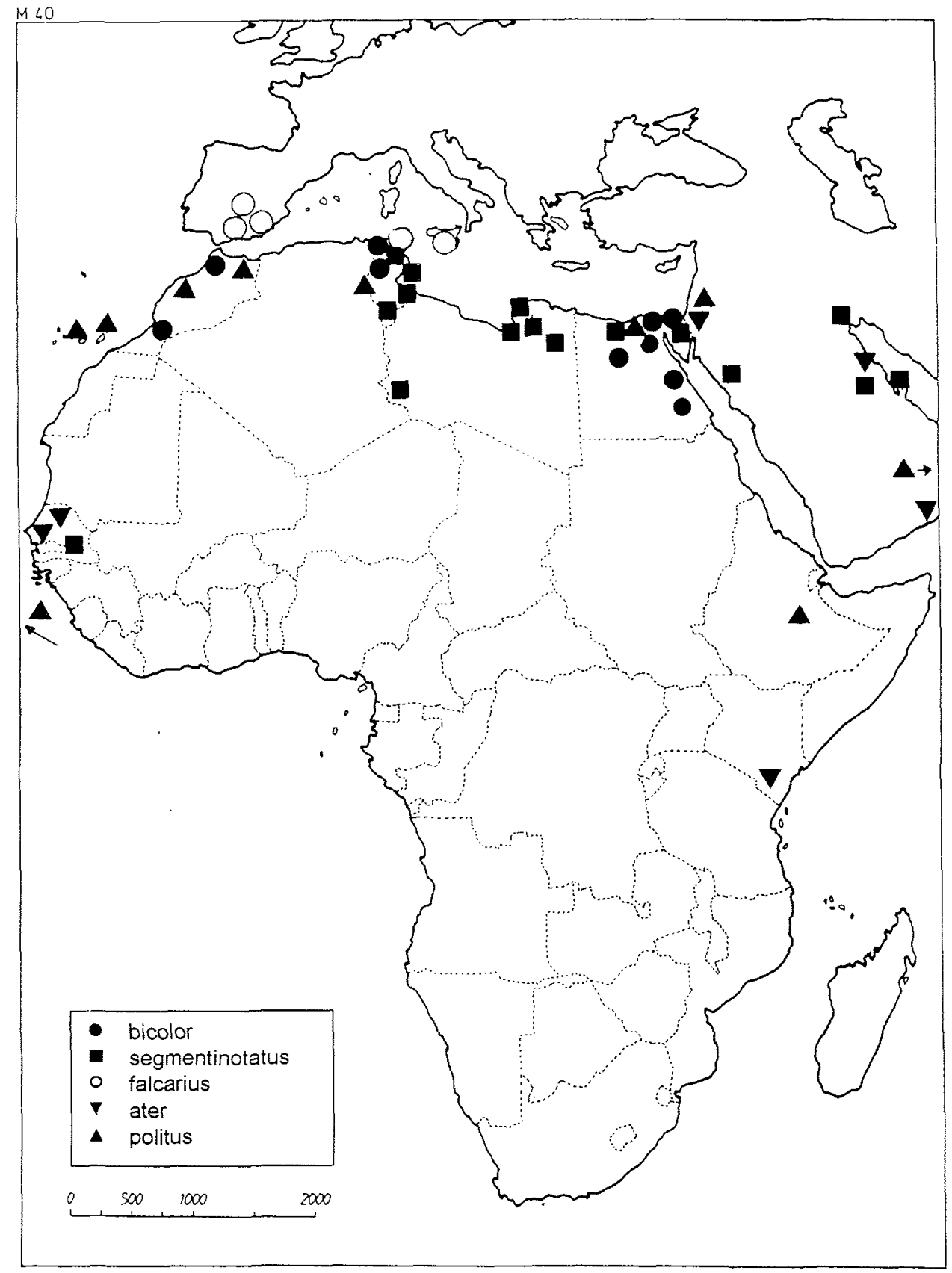

Fig. 10: Geographische Verbreitung der Gattung Enochrus (Lumetus) in Afrika. 


\section{B. Untergattung Enochrus s. str.}

\section{Bestimmungstabelle}

1 Sehr kleine Art, 2,5-3,0 mm; schwarz, Mesosternallamelle sehr niedrig, ohne apikales Zähnchen .................................................... E. pellax BALFOUR-BROWNE, 1958 Größere Arten, 4,2-5,5 mm, Oberseite fein bis mäßig grob punktiert .................. 2

2 Metafemora bis zu den Knien pubeszent. Mesosternallamelle hoch, mit Zähnchen vor der Mitte; Endglied der Maxillarpalpen apikal angedunkelt. Kurz oval, stark gewölbt, gelbbraun mit schwarzem Kopf und hellen Präokularmakeln ....

E. melanocephalus (OLIVIER, 1792)

Metafemora ohne Pubeszenz glatt. Mesosternallamelle flach ansteigend, mit Zähnchen etwa in der Mitte Länglich oval, weniger stark gewölbt; schwarz mit diffus aufgehellten Rändern; Elytren apikal mit 8 angedeuteten Punktreihen, [Guinea] E. mediastinus BALFOUR-BROWNE, 1959

\section{Die Arten}

\section{Enochrus (Lumetus) mediastinus BALFOUR-BROWNE, 1959}

1959 Enochrus (s. str.) mediastinus BALFOUR-BROWNE, p. 313 (Guinea: Kindia).

Locus typicus: Guinea.

Typenmaterial: 2 Männchen: Französ. Guinea, Kindia, 4.VI.1951 und 11.V.1951 (NMB, coll. Frey).

Diagnose: Oval, schwarz, lateral breit diffus aufgehellt; Oberseite dicht punktiert; Maxillarpalpen kaum länger als die Fühler; 3. und 4. Glied etwa gleichlang, Endglied apikal angedunkelt; Elytren subapikal mit 8 angedeuteten Punktreihen. Mesosternallamelle hoch, vorne schräg abfallend, dahinter flach auslaufend. Beine gelb; Metafemora völlig ohne Pubeszenz. Aedoeagus sehr ähnlich dem von E. picimus, wobei der bandförmige Medianlobus die Ventralplatte weit überragt (Fig. 4).

Diskussion: Der Autor selbst (BALFOUR-BROWNE, 1959) diskutiert die zweifelhafte systematische Stellung der Art aufgrund der Besonderheiten in der Pubeszenz der Mesound Metafemora sowie der angedeuteten 8 Punktreihen im apikalen Teil der Elytren, welche sie einerseits in die Nähe der primitiveren Untergattung I Lolcophilydrus KNIZ, 1911 rückt. Durch die fehlende Pubeszenz der Femora gerät sie andererseits in die Nähe von Enochrella HANSEN, 1999. Arten der Untergattung Ilolcopbilydrus sind bisher nur aus Madagaskar und Ostasien bekannt, wobei neben der typischen Form mit durchlaufenden Punktreihen vom selben Autor schon ein Jahr zuvor aus Madagaskar eine Art ( $E$. curtus BALFOUR-BROWNE, 1958) mit ähnlich reduzierten Punktreihen beschrieben wurde. 
Im Übrigen sind auch beim Genus-Typ, dem mitteleuropäischen E. melanocephalus OLIVIER im apikalen Viertel der Elytren deutliche Punkreihen erkennbar, so dass dieses Merkmal als regulär für die Untergattung zu betrachten ist.

Verbreitung: Westafrika (Guinea).

Enochrus (s. str.) melanocephalus (OLIVIER, 1792)

1792 Hydropbilus melanocephalus OLIVIER, p. 127.

1829 Hydrophilus atricapillus STEPHENS, p. 131 (England).

Locus typicus: Frankreich (,environs de Paris").

Diagnose: Drost, et al. (1992); HEBAUER \& KLAUSNitzer (1998); LOHSE (1971). Aufgrund des kurz ovalen und hochgewölbten Habitus sowie der charakteristischen Färbung und der kurzen Palpen mühelos zu erkennende Art mit weiter Verbreitung in der Paläarktis.

Kurzbeschreibung: Mittelgroße (4,2-5,4 mm), kurz ovale, hochgewölbte, gelbbraune Art mit schwarzem Kopf und großen hellen Präokularmakeln sowie zwischen $4 \mathrm{im}$ Rechteck stehenden schwarzen Punktfleckchen und manchmal kräftig geschwärzter Mitte des Pronotum. Maxillarpalpen kurz und gedrungen, das 2. Glied fast gerade, 3. und 4. Glied gleichlang, letzteres apikal geschwärzt (Fig. 8f). Elytren hell, mit schwarzen Schulterfleckchen und transparenten Punktlinien. Punktierung der Oberseite mäßig fein, doch sehr dicht; lateral und apikal mit angedeuteten 8 Punktreihen. Suturalreihen nach vorne bis zur Scutellarreihe reichend. Unterseite und Beine schwarz. Mitte des Metasternum mit Längsfurche. Mesosternallamelle vorne wenig steil abfallend, mit scharfem Zähnchen etwa in der Mitte, dahinter leicht konvex verlaufend (Fig. 9f). Aedoeagus mit parallel endenden Parameren; der schmale Medianlobus überragt die Ventralplatte mäßig weit (Fig. 6).

Verbreitung: Paläarktis; Afrika: Algerien.

Enochrus (s. str.) pellax BALFOUR-BRoWNE, 1958

1958 Enocbrzs (Enochrus) pellax BALFOLR-BROWNE, p. 50. („French Guinea“).

Locus typicus: Guinea ("French Guinea").

Typenmaterial: s. HeBAUER (2001:389).

Nachweise: s. HEBAUER (2001: 389). 


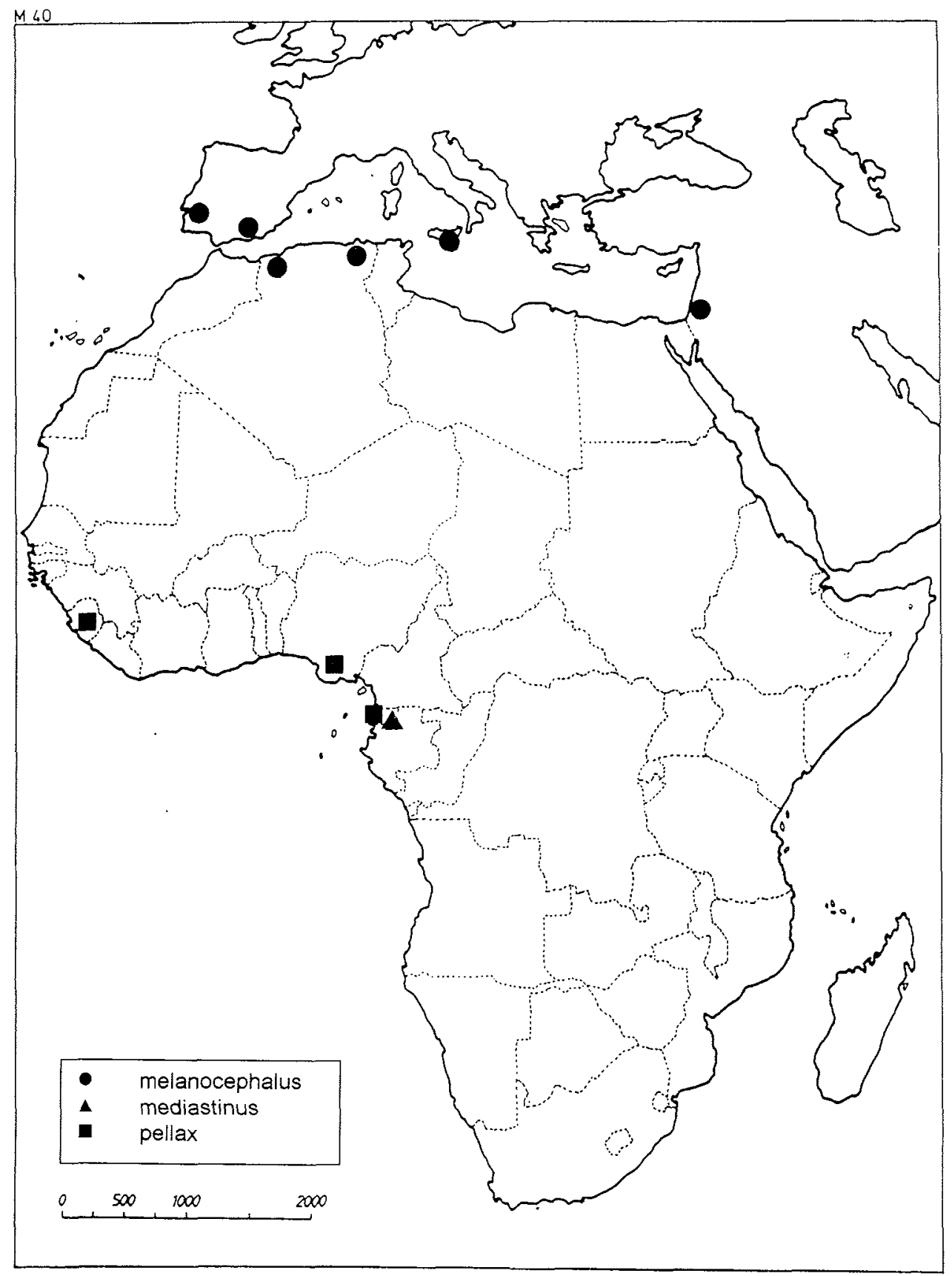

Fig. 11: Geographische Verbreitung der Gattung Enochrus (s. str:) in Afrika. 
Diagnose: Wegen der Kleinheit und untypischen Merkmale eher mit einer MethydrusArt als einem Enocbrus s. str. zu verwechseln.

Kurzbeschreibung: Sehr kleine (2,5-3,0 mm) einfarbig glänzend pechschwarze, länglich ovale und hochgewölbte Art ohne Präokularmakeln; lediglich die Seitenränder von Pronotum und Elytren sind schmal gelb aufgehellt. Die Maxillarpalpen sind relativ (für Enochrus s. str. untypisch) lang und schlank, das 2. Glied außen deutlich konkav, das Endglied kaum kürzer als das vorletzte Glied und völlig schwarz (Fig. 13h in HEBAUER, 2001). Kopf und Pronotum fein, etwas locker, Elytren deutlich kräftiger und dichter punktiert. Beine kastanienbraun. Mesosternallamelle sehr flach bogig, ohne Zähnchen (!). Analsternit apikal nicht ausgerandet. Aedoeagus mit basal sehr breiten, im distalen Drittel stark verjüngten, gerade auslaufenden Parameren. Medianlobus viel kürzer als die Parameren, mäßig breit bandförmig, apikal kurz verrundet, die Ventralplatte sehr weit überragend. (Fig. 1 in HEBAUER, 2001).

Die umstrittene bzw zweideutige systematische Stellung der Art (Metbydus / Enochrus) wurde aus praktischen Gründen bereits im 2. Teil der „Taxonomischen Studien ...“ (HEBAUER, 2001) in die Bestimmungstabelle integriert.

Verbreitung: Westafrika (Guinea, Nigeria, Sierra Leone).

\section{Literatur}

BALFOUR-Browne, J. 1951: Coleoptera: Haliplidae, Dytiscidae, Gyrinidae, Hydraenidae, Hydrophilidae (pp. 179-220, pl. 10-11). - In: British Museum (Natural History): Expedition to South-west Arabia 19371938. xiv + 504 pp. - London.

BALFour-Browne, J. 1958: New species of Malgassic Hydrophilidae (Col.). - Mauritius Institute Bulletin, 5: $134-147$.

BAI_Four-BrowNe, J. 1959: Dr. Jan Bechyné expedition to French Guinea, 1951. Hydtophilidae. - Entomologische Arbeiten aus dem Museum G. Frey, 10: 302-320.

BLANCHARD, E. 1853: Description des Insectes (= Zoologie, vol. 4). - In: Voyage au Póle Sud et dans 1' Océanie sur les corvettes l'Astrolabe et la Zélée; exécuté par ordre du roi pendant les années 1837-18381839-1840, sous le commandement de M.J. Dumont-d'Urville, capitaine de vaisseau ... 422 pp. - Gide et $J$. Baudry, Paris.

Drost, M. B. P. \& Cuppen, H. P. J. J. \& VAN Nieukerken, E. J. \& SChreijer, M. 1992: De Waterkevers van Nederland. - Stichting Uitgeverij Koninklijke Nederlandse Natuurhistorische Vereniging. Nationaal Natuurhistorisch Museum; 280 pp.

Fabricius, J. C. 1787: Mantissa Insectorum ... Vol. $1.20+348$ pp. - C. G. Proft, Hafniae.

FABRICIUS, J. C. 1792: Entomologia Systematica ... Vol. $1.20+330$ pp. - C. G. Proft, Hafniae.

FaIRMaIRE, L. 1879: Descriptions des Coléoptères nouveaux du nord de l'A frique. - Annales de la Société entomologique de France, (5) 9: 155-172.

GYLLENHAL, L. 1827: Insecta Suecica ... Vol. 1, pars 4.762 pp. - F. Fleischer, Lipsiae.

HANSEN, M. 1999: Fifteen new genera of Hydrophilidae (Coleoptera), with remarks on the generic classification of the family. - Entomologica scandinavica, Copenhagen 30:121-172.

HebAler, F. 1989: 9. Familie Hydrophilidae. - In: G. A. Lohse and W. H. Lucht (eds.): Die Käfer Mitteleuropas. 1. Supplementband. Krefeld. Goecke \& Evers, pp. 82-92.

HEBAUER, F. 1991: Enocbrus (Limetus) falcarius n. sp., aus Sizilien (Coleoptera, Hydrophilidae). - Acta coleopterologica, 7 (2): 83-86. 
HeBAUER, F. \& KLAUSNTtzer, B. 1998: Insecta: Coleoptera: Hydrophiloidea (exkl. Helophorus). Süßwasserfauna von Mitteleuropa, 20/7, 8, 9, 10-11, 134 pp. Gustav Fischer.

HEBAUER, F. 1999: Neue und wenig bekannte Hydrophiloidea aus dem südlichen Afrika (Coleoptera, Hydrophiloidea). - Acta coleopterologica, 15 (2): 7-17.

HebaUer, F. 2001: Taxonomische Studien zur Hydrophiliden-Gattung Enochrms Thomson, 1859. 2. Teil: Die afrikanischen Arten der Untergattung Metbydrus REY, 1885. A: Die Enoclmus meracus-Gruppe. (Coleoptera, Hydrophilidae). - Beiträge zur Entomologie, Keltern 51 (2): 375-391.

Hernando, C.; Riberi, L.; Aguileri, P. \& FRESNEDil, J. 1997: Enochms falcarins HeBiUer, 1991 new for continental Europe (Coleoptera, Hydrophilidae). - Nouvelle Revue d'Entomologie (Nouvelle Setie), 14 (2): 133-134.

KÜSTER, H. C. 1849 (1844-55): Die Käfer Europa's. Nach der Natur beschrieben. Heft 18. 4 (unn.) pp. +100 Blatt, 2 pl. - Bauer \& Raspe, Nürnberg.

KUwerT, A, 1888: Generalübersicht der Philydrus-Artet Eutopas und der Mittelmeerfauna. - Deutsche entomologische Zeitschrift, 32: 273-293.

KUWERT, A. 1890: Bestimmungs-Tabellen der europäischen Coleopteren. XIX. Heft. Hydtophilidae. I. Abteilung Hydrophilini. - Verhandlungen des naturforschenden Vereins in Brünn, 28 (1889): 1-121.

LOHSE, G. A. 1971: Familienreine Palpicornia (Wasserkäfer, Wassetfreunde) (Hydraenidae, Spercheidae, Hydrophilidae). - In: Fredde, H. \& HARDE, K. W. \& LOHSE, G. A.: Die Käfer Mitteleuropas, Bd. 3: 95156. Krefeld.

MARSHAM, T. 1802: Entomologia Brittannica ... Vol. 1. Coleoptera. 31 + 548 pp. - Wilks et Taylor, London. REY, C. 1885: Histoire naturelle des Coléoptères de France (suite). - Annales de la Société linnéenne de Lyon 31 (1884): 213-396.

Matsui, E. 1994: Three new species of the genus Enoibrus from Japan and Taiwan (Coleoptera: Hydtophilidae). - Transactions of the Shikoku entomological Society, 20:215-220.

ROTTENBERG, A. von, 1870: Beiträge zur Coleopteren-Fauna von Sizilien. - Berliner entomologische Zeitschrift, 14: 11-40.

OLmier, A. G. 1792: Encyclopédie méthodique. Dictionnaire des Insectes. Vol. 7 (Han-Mou). 827 pp. Pankouke, Paris. (Pl. 183, with illustrations of Hydrophilus, issued in 1797).

SAHLBERG, J. 1900: Coleoptera mediterranea et rosso-asiatica vel minus cognita itineribus annis 1895-1896 et 1898-1899 collecta. - Öfversigt av finska Vetenskabssocietetens Förhandlingar, 42: 174-208.

ScHÖDL, S. 1998: Taxonomic revision of Enochrus (Coleoptera: Hydrophilidae) I. The E. bicolor species complex. - Entomological Problems, 29 (2): 111-127.

StEPHENS. J. F. 1829 (1828-1829): Illustrations of British Entomology ... Mandibulata. Vol. 2, 200 pp., pl. 10-15. - Baldwin and Cradock, London.

THOMSON, C. G. 1853: Offersigt af de i Sverige funna arter af familjen Palpicornia. - Öfersigt af K. Vetenskabsakademiens Förhandlingar, 10: 40-58.

Thomson, C. G. 1859: Skandinaviens Coleoptera, Vol. 1, 290 pp. Berlingska Boktryckeriet, Lund.

ZAITZEV, F. A. 1908: Catalogue des Coléoptères aquatiques des familles Dryopidae, Georyssidae, Cyathoceridae, Heteroceridae et Hydtophilidae. - Horae Societatis entomologicae rossicae 38: 283-420.

\section{Anschrift des Verfassers:}

Dr. habil. Franz HeBALER

Ulrichsberg 7

D-94539 Grafling 